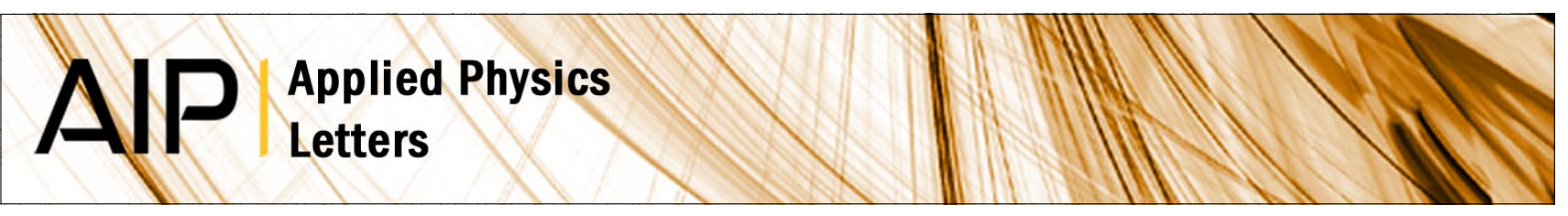

\title{
Structure of droplet-epitaxy-grown InAs/GaAs quantum dots
}

Eyal Cohen, Shira Yochelis, Ohad Westreich, Sergey Shusterman, Divine P. Kumah et al.

Citation: Appl. Phys. Lett. 98, 243115 (2011); doi: 10.1063/1.3599063

View online: http://dx.doi.org/10.1063/1.3599063

View Table of Contents: http://apl.aip.org/resource/1/APPLAB/v98/i24

Published by the AIP Publishing LLC.

Additional information on Appl. Phys. Lett.

Journal Homepage: http://apl.aip.org/

Journal Information: http://apl.aip.org/about/about_the_journal

Top downloads: http://apl.aip.org/features/most_downloaded

Information for Authors: http://apl.aip.org/authors

\section{ADVERTISEMENT}
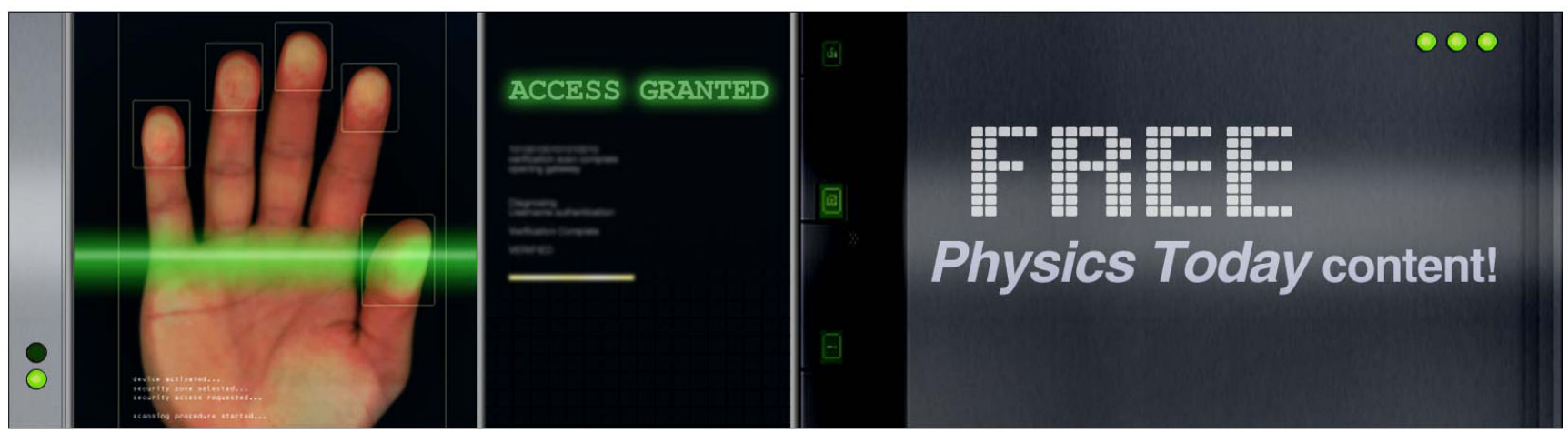


\title{
Structure of droplet-epitaxy-grown InAs/GaAs quantum dots
}

\author{
Eyal Cohen, ${ }^{1}$ Shira Yochelis, ${ }^{1}$ Ohad Westreich, ${ }^{1,2}$ Sergey Shusterman, ${ }^{2}$ \\ Divine P. Kumah, ${ }^{3, a)}$ Roy Clarke, ${ }^{3}$ Yizhak Yacoby, ${ }^{4}$ and Yossi Paltiel ${ }^{1, b)}$ \\ ${ }^{1}$ Department of Applied Physics, Hebrew University, Jerusalem 91904, Israel \\ ${ }^{2}$ Solid State Physics, Electro-Optics Division, Soreq NRC, Yavne 81800, Israel \\ ${ }^{3}$ Applied Physics Program, University of Michigan, Ann Arbor, Michigan 48109, USA \\ ${ }^{4}$ Racah Institute of Physics, Hebrew University, Jerusalem 91904, Israel
}

(Received 10 April 2011; accepted 20 May 2011; published online 17 June 2011)

\begin{abstract}
We have used a direct $\mathrm{x}$-ray phasing method, coherent Bragg rod analysis, to obtain sub-angstrom resolution electron density maps of the InAs/GaAs dot system. The dots were grown by the droplet heteroepitaxy (DHE) technique and their structural and compositional properties are compared with those of dots grown by the strain-driven Stranski-Krastanov method. Our results show that the Ga diffusion into the DHE-grown dots is somewhat larger; however, other characteristics such as the composition of the dots' uppermost layers, the interlayer spacing, and the bowing of the atomic layers are similar. (C) 2011 American Institute of Physics. [doi:10.1063/1.3599063]
\end{abstract}

Self-assembled semiconductor quantum dots (QDs) are of current interest because their optoelectronic properties can be controlled by tailoring their size, shape and composition. Located in size between isolated atoms and bulk material, QDs act as "artificial atoms" with electrons and holes confined in all three spatial dimensions. These unique quantum properties have attracted a great deal of scientific and technological interest in recent years, in the fields of optoelectronic devices, ${ }^{1,2}$ quantum information processing, ${ }^{3}$ and energy harvesting. ${ }^{4-6}$

Strain-driven growth methods, based on the StranskiKrastanov (SK) mechanism, are commonly used to fabricate self-assembled QDs. ${ }^{7-9}$ This mechanism requires a large lattice mismatch between the substrate and the dot material, and surface energy dominates the dots' shape.

This constrains the combinations of materials that can be used to form QDs by the SK approach. ${ }^{10,11}$ A more recent technique for QD fabrication, the droplet heteroepitaxy (DHE) method, ${ }^{12,13}$ has no such limitations. It has been demonstrated $^{14}$ that the DHE method is more relaxed in the combination of materials that can be used, allowing great flexibility in realizing high density QDs grown on almost any substrate. This method consists of two basic steps: first, for III-V QDs, nanodroplets of the group III element are formed on the substrate. Subsequently, these droplets are exposed to a gas phase flow of the group $\mathrm{V}$ element. Although the growth process starts with liquid droplets, in the end, under proper conditions, the formed dots are single crystals, atomically registered with the underlying substrate.

Previous work has demonstrated that the DHE dot growth is very sensitive to the process parameters. ${ }^{12,15}$ The mechanisms taking part in the QD formation are quite complex ${ }^{14}$ and are not yet fully understood. Strong material intermixing, ${ }^{16}$ strain, and dislocations ${ }^{17}$ have all been observed. Consequently, detailed knowledge of the structural and compositional properties of the system is essential for understanding the growth process.

In this letter, we show the results of x-ray diffraction measurements performed on the well known system of InAs/

\footnotetext{
${ }^{a)}$ Present address: CRISP, Yale University, New Haven, CT.

${ }^{b)}$ Electronic mail: paltiel@cc.huji.ac.il.
}

GaAs QDs. This system was chosen for two main reasons: first, simplicity; the system contains only 3 elements: In, Ga, and As. Second, the equivalent QD system grown by the SK method has been widely studied. ${ }^{18-21}$

The QDs for this study were grown in a metallorganic vapor phase reactor. The growth procedure is described in detail elsewhere. ${ }^{13}$ This process is similar to the one used previously to grow such dots, ${ }^{16}$ but with temperatures lower by $20{ }^{\circ} \mathrm{C}$ during the process. The lower temperature allowed us to obtain smaller, denser dots. ${ }^{14}$

Figure 1(a) (inset) shows an atomic force microscopy (AFM) image of the InAs QDs sample under investigation. The height of the dots is about $5 \mathrm{~nm}$, with lateral widths

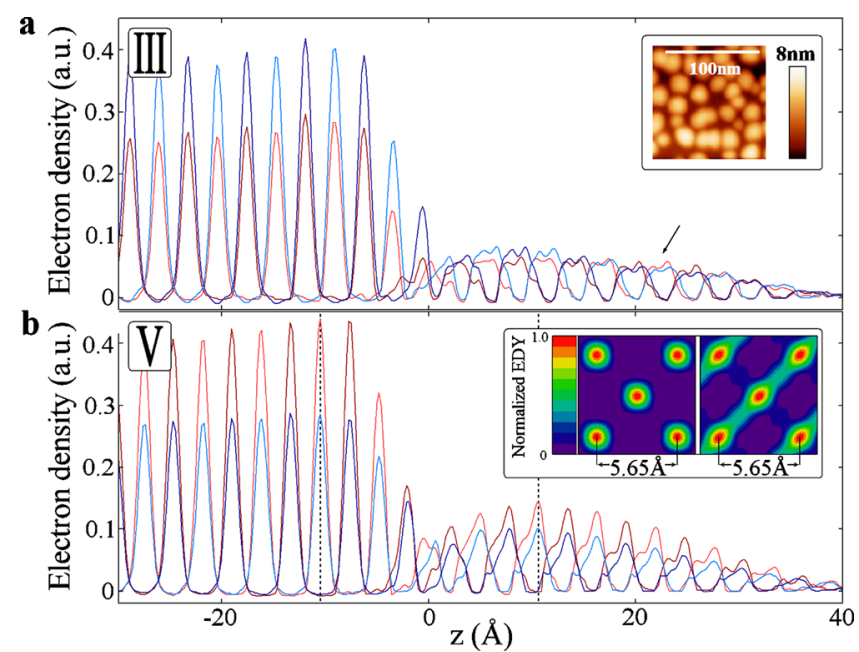

FIG. 1. (Color online) EDY profiles along (001) lines going through the center positions of (a) group III ( $\mathrm{Ga}$ ) and (b) group V (As) atoms in the substrate. The lines corresponding to smaller peaks in (a) and larger peaks in (b) were measured at the Ga K-edge X-ray energy, while the larger peaks in (a) and smaller peaks in (b) correspond to the As K-edge X-ray energy. The lighter and darker profiles correspond to two different (001) lines that go through two different atoms of the same species in the substrate. The arrow in (a), around $\mathrm{z}=23 \AA$, marks the approximate onset location of pure InAs. This is based on the calculation of the In concentration using Eq. (1) and the 3D integrated EDYs. Inset (a) shows a topographic AFM image of the InAs QDs formed on the GaAs substrate. Inset (b) shows the EDY on two planes: one in the substrate the other in the dots, the positions of both marked by the dotted lines in (b). 
ranging between 10 and $40 \mathrm{~nm}$. The areal density of the QDs, as inferred from AFM data and from high resolution scanning electron microscopy (SEM) images, is higher than $10^{11} / \mathrm{cm}^{2}$. All dots have an oval-like cross-section with a contact angle of approximately $120^{\circ}$, as revealed by high resolution SEM measurements performed in a tilted-sample geometry.

We have used a direct nondestructive x-ray phasing method known as coherent Bragg rod analysis (COBRA) (Refs. 22 and 23) to study the structure and composition of the dots in a way similar to other recently studied QD systems. ${ }^{16}$ COBRA uses the measured diffraction intensities along the substrate-defined Bragg rods to provide the folded structure of the QD system. By the folded structure we mean the structure obtained when each atom in the system is translated to one substrate-defined 2D unit cell using the substrate-defined 2D unit cell vectors. ${ }^{22}$ Diffraction intensities are received from dots in a $1 \mathrm{~mm} \times 1 \mathrm{~mm}$ spot size, averaged from coherent areas of around $10 \mu \mathrm{m}^{2}$ in size. By collecting the diffraction data for each rod at two beam energies-just below the $\mathrm{Ga} \mathrm{K}$-edge $\left(\mathrm{E}_{1}=10.362 \mathrm{keV}\right)$ and just below the As K-edge $\left(E_{2}=11.864 \mathrm{keV}\right)$-the difference between the scattering factors of the two elements is maximized. Comparing the effective electron density (EDY) obtained at the two energies enables us to determine the concentrations of $\mathrm{Ga}$ and $\mathrm{In}$ atoms in the folded structure.

Figure 1 displays the EDY profiles along (001) lines passing through the centers of group III and group $\mathrm{V}$ atoms in the zinc-blende structure, obtained using the two x-ray beam energies. In inset $\mathrm{b}$ we show the EDY of the folded structure on two planes parallel to the surface at positions marked by the dotted lines in Fig. 1(b). One plane is in the substrate, the other in the dots. The EDYs in both cases are atomic like. The EDY between the peaks along the (110) direction are tails of the atoms in the layer above. These results show that the growth is epitaxial.

From Fig. 1, we find that the interlayer spacing in the substrate and in the dots are equal to within 3\%. This result is surprising because the unit cell of InAs is larger than that of GaAs by about $6 \%$. Moreover, the substrate applies lateral compressive stress on the dot which would tend to further increase the vertical spacing. This behavior has also been observed in the SK grown dot system ${ }^{24}$ and was ascribed to bowing of the atomic layers in the QD.

The relative concentration of In $\rho_{\mathrm{In}} /\left(\rho_{\mathrm{In}}+\rho_{\mathrm{Ga}}\right)$ in the dot structure can be calculated by using the effective three dimensionally integrated EDYs at the Ga/In sites determined at the two x-ray energies, $Q_{\mathrm{Ga}}$ and $Q_{\mathrm{As}}$

$$
\frac{\rho_{\mathrm{In}}}{\rho_{\mathrm{Ga}}+\rho_{\mathrm{In}}}=\frac{\sigma_{\mathrm{As}}^{\mathrm{Ga}} Q_{\mathrm{Ga}} / Q_{\mathrm{As}}-\sigma_{\mathrm{Ga}}^{\mathrm{Ga}}}{\left(\sigma_{\mathrm{As}}^{\mathrm{Ga}}-\sigma^{\mathrm{In}}\right) Q_{\mathrm{Ga}} / Q_{\mathrm{As}}+\sigma^{\mathrm{In}}-\sigma_{\mathrm{Ga}}^{\mathrm{Ga}}},
$$

where $\sigma_{\mathrm{Ga}}^{\mathrm{Ga}}$ and $\sigma_{\mathrm{As}}^{\mathrm{Ga}}$ are the scattering cross sections of $\mathrm{Ga}$ at the x-ray photon energies just below the Ga and As K-edges, respectively and $\sigma^{\mathrm{In}}$ is the scattering cross-section of In at both edges (practically constant at these energies).

The occupancy results are presented in Fig. 2(a). They show that some Ga diffuses from the substrate into the dots. We believe that the Ga diffusion takes place already when the In droplets are deposited. This mixing forms a layer of InGaAs with a gradual decrease in Ga concentration as we move higher into the dot. Above a height of approximately $2.3 \mathrm{~nm}$ (corresponding to about 4 unit cells), measured from
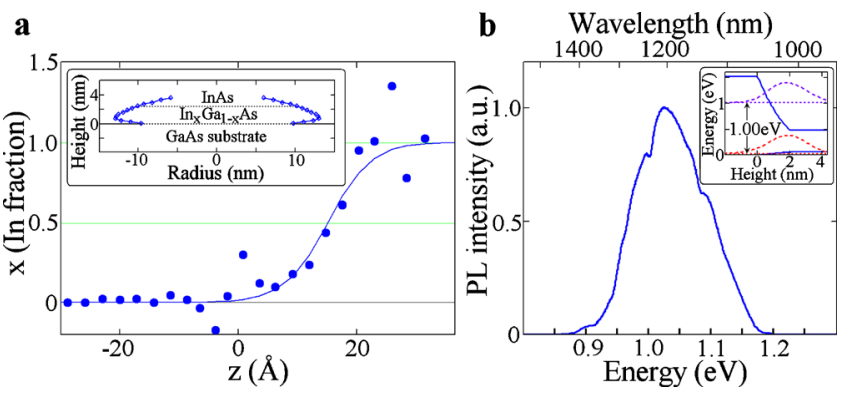

FIG. 2. (Color online) (a) Fractional In occupancy on lateral atomic layers as a function of the layer's distance from the nominal interface. Inset shows a diagram of the dot profile calculated from the EDY maps. Note the large contact angle of the dots to the substrate, which is ascribed to the nonwetting process of the In droplets. (b) PL spectrum, measured at $10 \mathrm{~K}$. Insetone-dimensional diagram of the hole and electron density distributions in the dot (dashed). The solid curves show the nominal valence (lower curve) and conduction (upper curve) band edges of $\operatorname{In}_{\mathrm{x}} \mathrm{Ga}_{1-\mathrm{x}} \mathrm{As}$ as a function of the distance from the interface. The spacing between the dotted lines represents the hole electron energy difference.

the nominal substrate/dot interface, we see only InAs. For comparison, Ga penetrates only about 2 unit cells into the SK grown dot system. It is also evident from the present results that the "nanodrilling" of the dots into the substrate, observed previously in the GaAs-InSb system, ${ }^{16}$ is absent here and neither was it observed in the SK grown sample. The reason for this different behavior is not clear; it may relate to the role of Sb acting as a surfactant. ${ }^{25,26}$

Since COBRA provides us with the fill factor of each layer, by knowing the dots' average shape (using AFM/SEM measurements) one can calculate the density of the dots. Figure 2(a) inset shows profile of the dots, calculated from the obtained fill factor and the dots known density. Note the large contact angle which we ascribe to the In nonwetting conditions at the first stage of the growth.

It is interesting to notice that in all substrate-like EDY peaks the effective EDY measured with As edge $\mathrm{x}$-rays is larger than that measured with Ga edge X-rays by about the ratio of $\sigma_{\mathrm{As}}^{\mathrm{Ga}} / \sigma_{\mathrm{Ga}}^{\mathrm{Ga}}$, meaning that the InAs does not form a continuous layer. This is expected in the DHE growth method. A wetting layer was also absent in the SK grown sample. The absence was ascribed to the wetting layer being consumed by the dots when the areal density is very high. ${ }^{24}$

Photoluminescence (PL) measurements at $10 \mathrm{~K}$, presented in Fig. 2(b), show a peak centered at about $1 \mathrm{eV}$. The PL signal is wide due to the large spread in dot sizes. PL is known to be sensitive to many factors such as chemical intermixing, quantum confinement, strain and piezoeffects. Therefore, using the COBRA information in order to obtain a correct PL spectrum is hard to achieve. However, we can make a rough estimate of the PL peak energy by calculating the electron-excitation energy in a quantum well (diameter $\gg$ height) with the average dot composition profile shown in Fig. 2(a). The calculation was done using the NEXTNANO (Ref. 27) program and the result obtained is $1 \mathrm{eV}$, [shown in Fig. 2(b) inset] consistent with the experimental result.

In our dot system, the EDY peaks in the dots region reveal a number of additional interesting features: (1) the shape of each EDY peak is broadened and appears to have a quasi-rectangular shape. This is ascribed to the bowing of the atomic layers in the dot. ${ }^{24}$ (2) The EDY peaks of both group III and group V elements appear to be split and shifted relative to the layer positions extrapolated from the substrate 

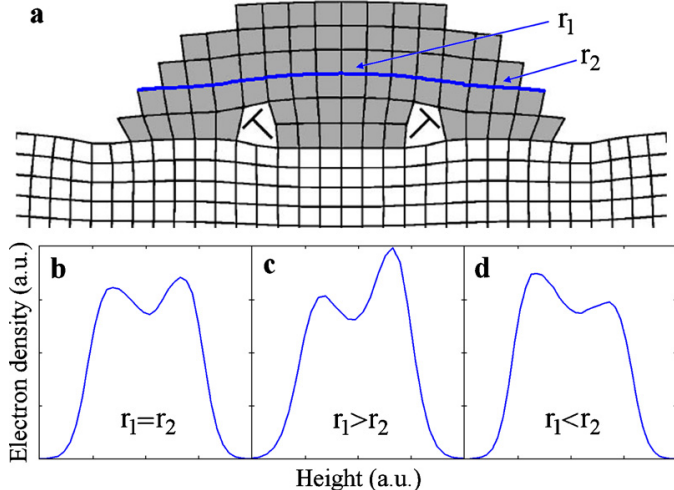

FIG. 3. (Color online) (a) Deformations in the epitaxial island, taken from Ref. 8. $\mathrm{r}_{1}$ represents the radius of curvature of the curved region between the dislocations and $r_{2}$ that of the curved region on both side of the pair of dislocations. Folding a single atomic layer of the dot (solid line) into a 2D substrate-defined unit cell and taking the EDY along a line going through the maximum yields the profiles seen in (b)-(d).

[see Figs. 1(a) and 1(b), respectively]. This splitting is small and is close to the uncertainty limit, yet it is also observed in the SK grown sample where the behavior was described as interstitial stacking fault shift. ${ }^{24}$ Moreover, error analysis using simulated noise ${ }^{28}$ shows that the peak splitting is quite robust.

The shift from the nominal atomic positions and the peak splitting occur already at the first monolayer of the QDs. This indicates that this phenomenon originates at the interface between the substrate and the dot. Previous high resolution transmission electron microscopy scans taken on buried III-V DHE QDs have revealed edge dislocations at the interface between the substrate and the dot. ${ }^{29}$ The shape of our dots matches the shape of such buried dots obtained by TEM and cross-sectional STM scans. Spencer and Tersoff $^{8}$ mapped the strain in the dots taking into account these dislocations and the unit cell mismatch between the substrate and the dots. We can consider each layer to be approximately composed of two bowed regions with radii of curvature $r_{1}$ and $r_{2}$. This is shown in Fig. 3(a). Folding the resulting structure into one substrate-defined 2D unit cell and taking the EDY along the (001) direction yields the EDY profiles shown in Figs. 3(b) and 3(d) for $r_{1}=r_{2}, r_{1}>r_{2}, r_{1}$ $<\mathrm{r}_{2}$, respectively. Notice that the shapes are qualitatively similar to the experimentally observed EDY peak shapes shown in Figs. 1(a) and 1(b).

Diffusion of In to interstitial sites in the zinc-blende structure $^{30,31}$ is another process which might be considered as a possible mechanism to explain the EDY splitting. Yet such a phenomenon should only have a minor effect, considering the low concentration of interstitial defects in the crystal.

To summarize, we have revealed the atomic structure and composition of InAs/GaAs QDs grown by the DHE method. Comparison between these dots and those grown by the SK method using MBE shows that the Ga diffusion into the dots is somewhat larger in the DHE grown dots. We note that interdiffusion depends strongly on temperature and therefore this difference will depend on specific growth parameters. Finally, the atomic layers in both systems are bowed and the center positions are shifted relative to an extrapolation of the substrate layer sequence.

This work was supported by the U.S. National Science Foundation under Grant No. DMR-0906909. Use of the Advanced Photon Source, an Office of Science User Facility operated for the U.S. Department of Energy (DOE) Office of Science by Argonne National Laboratory, was supported by the U.S. DOE under Contract No. DE-AC02-06CH11357.

${ }^{1}$ A. D. Yoffe, Adv. Phys. 50, 1 (2001).

${ }^{2}$ N. N. Ledentsov, V. M. Ustinov, V. A. Shchukin, P. S. Kop'ev, Z. I. Alferov, and D. Bimberg, Semiconductors 32, 343 (1998).

${ }^{3}$ E. Biolatti, R. C. Iotti, P. Zanardi, and F. Rossi, Phys. Rev. Lett. 85, 5647 (2000).

${ }^{4}$ V. Aroutiounian, S. Petrosyan, A. Khachatryan, and K. Touryan, J. Appl. Phys. 89, 2268 (2001).

${ }^{5}$ M. Grätzel, Nature (London) 414, 338 (2001).

${ }^{6}$ I. Robel, V. Subramanian, M. Kuno, and P. V. Kamat, J. Am. Chem. Soc. 128, 2385 (2006).

${ }^{7}$ R. Nötzel, J. Temmyo, and T. Tamamura, Nature (London) 369, 131 (1994).

${ }^{8}$ B. J. Spencer and J. Tersoff, Phys. Rev. B 63, 205424 (2001).

${ }^{9}$ G. Springholz, V. Holy, M. Pinczolits, and G. Bauer, Science 282, 734 (1998)

${ }^{10}$ S. Fafard, Z. R. Wasilewski, and M. Spanner, Appl. Phys. Lett. 75, 1866 (1999).

${ }^{11}$ B. Lita, R. S. Goldman, J. D. Phillips, and P. K. Bhattacharya, Appl. Phys. Lett. 75, 2797 (1999).

${ }^{12}$ N. Koguchi, K. Ishige, and S. Takahashi, J. Vac. Sci. Technol. B 11, 787 (1993).

${ }^{13}$ S. Shusterman, Y. Paltiel, A. Sher, V. Ezersky, and Y. Rosenwaks, J. Cryst. Growth 291, 363 (2006).

${ }^{14}$ S. Shusterman, A. Raizman, and Y. Paltiel, Infrared Phys. Technol. 52, 229 (2009).

${ }^{15}$ K. A. Sablon, J. H. Lee, Z. M. Wang, J. H. Shultz, and G. J. Salamo, Appl. Phys. Lett. 92, 203106 (2008).

${ }^{16}$ D. P. Kumah, S. Shusterman, Y. Paltiel, Y. Yacoby, and R. Clarke, Nat. Nanotechnol. 4, 835 (2009).

${ }^{17}$ S. Shusterman, A. Raizman, A. Sher, Y. Paltiel, A. Schwarzman, E. Lepkifker, and Y. Rosenwaks, Nano Lett. 7, 2089 (2007).

${ }^{18}$ L. W. Wang, J. N. Kim, and A. Zunger, Phys. Rev. B 59, 5678 (1999).

${ }^{19}$ D. M. Bruls, J. W. A. M. Vugs, P. M. Koenraad, H. W. M. Salemink, J. H. Wolter, M. Hopkinson, M. S. Skolnick, F. Long, and S. P. A. Gill, Appl. Phys. Lett. 81, 1708 (2002).

${ }^{20}$ M. Grundmann, O. Stier, and D. Bimberg, Phys. Rev. B 52, 11969 (1995).

${ }^{21}$ P. B. Joyce, T. J. Krzyzewski, G. R. Bell, B. A. Joyce, and T. S. Jones, Phys. Rev. B 58, R15981 (1998).

${ }^{22}$ Y. Yacoby, R. Pindak, R. MacHarrie, L. Pfeiffer, L. Berman, and R. Clarke, J. Phys.: Condens. Matter 12, 3929 (2000).

${ }^{23}$ M. Sowwan, Y. Yacoby, J. Pitney, R. MacHarrie, M. Hong, J. Cross, D. A. Walko, R. Clarke, R. Pindak, and E. A. Stern, Phys. Rev. B 66, 205311 (2002).

${ }^{24}$ D. P. Kumah, J. H. Wu, N. S. Husseini, V. D. Dasika, R. S. Goldman, Y. Yacoby, and R. Clarke, Appl. Phys. Lett. 98, 021903 (2011).

${ }^{25}$ J. C. Harmand, L. H. Li, G. Patriarche, and L. Travers, Appl. Phys. Lett. 84, 3981 (2004).

${ }^{26}$ C. S. Peng, Q. Huang, W. Q. Cheng, J. M. Zhou, Y. H. Zhang, T. T. Sheng, and C. H. Tung, Appl. Phys. Lett. 72, 2541 (1998).

${ }^{27}$ S. Birner, T. Zibold, T. Andlauer, T. Kubis, M. Sabathil, A. Trellakis, and P. Vogl, IEEE Trans. Electron Devices 54, 2137 (2007).

${ }^{28}$ H. Zhou, R. Pindak, R. Clarke, D. Steinberg, and Y. Yacoby, (unpublished).

${ }^{29}$ S. Shusterman, A. Raizman, A. Sher, A. Schwarzman, O. Azriel, A. Boag, Y. Rosenwaks, P. L. Galindo, and Y. Paltiel, EPL 88, 66003 (2009).

${ }^{30}$ I. C. Kizilyalli, T. L. Rich, F. A. Stevie, and C. S. Rafferty, J. Appl. Phys. 80, 4944 (1996).

${ }^{31}$ D. A. Antoniadis and I. Moskowitz, J. Appl. Phys. 53, 9214 (1982). 